KRZYSZTOF BuRnECKI (Wrocław)

Grzegorz KuKLa (Wrocław)

\title{
PRICING OF ZERO-COUPON AND COUPON CAT BONDS
}

Abstract. We apply the results of Baryshnikov, Mayo and Taylor (1998) to calculate non-arbitrage prices of a zero-coupon and coupon CAT bond. First, we derive pricing formulae in the compound doubly stochastic Poisson model framework. Next, we study 10-year catastrophe loss data provided by Property Claim Services and calibrate the pricing model. Finally, we illustrate the values of the CAT bonds tied to the loss data.

1. Introduction. Catastrophe bonds, also called "CAT bonds", are insurance-linked securities that enable insurers to transfer the risk of natural disasters like earthquakes or hurricanes to capital markets. They are sold to large institutions. A financial intermediary, a reinsurance company or an investment bank, issues a bond to a particular insurable event like e.g. Los Angeles earthquake. The proceeds from the sale are put into a collateral account. If there is no earthquake, investors are paid generous interest rate, but if the earthquake occurs and the claims exceed a specified amount, investors sacrifice fully or partially their principal and interest.

For insurers the deals create a pool of money that can be tapped immediately into a disaster. CAT bonds appeal to professional money managers because catastrophe risk is a new asset class that is uncorrelated with stocks and traditional bonds. They are growing in importance also because insurance capacities worldwide have been severely reduced by the events of 11 September 2001.

In the article of Baryshnikov et al. (1998) the authors presented an arbitrage-free solution to the pricing of CAT bonds under conditions of continuous trading. They modelled the stochastic process underlying the CAT bond as a compound doubly stochastic Poisson process. In Section 2

2000 Mathematics Subject Classification: 62P05, 62-07, 60G55, 60H30.

Key words and phrases: catastrophe bond, doubly stochastic Poisson process, loss distribution, non-arbitrage price, non-parametric test. 
we apply their results to calculate non-arbitrage prices of a zero-coupon and coupon CAT bond. In Section 3 we study 10-year catastrophe loss data provided by Property Claim Services (PCS). We find a distribution function which fits the observed claims in a satisfactory manner and estimate the intensity of the Poisson process governing the flow of the natural events. In Section 4 we illustrate the values of the CAT bonds associated with the loss data with respect to the threshold level and maturity time. To this end we apply Monte Carlo simulations.

2. Compound doubly stochastic Poisson pricing model. The CAT bond price process $V_{t}, t \in[0, T]$, is modelled by many factors: type of region, kind of loss event, sort of insured property etc. Baryshnikov et al. (1998) describe the bond by means of the aggregate loss process $L_{t}$ and the trigger value $D$. Set a probability space $\left(\Omega, \mathcal{F}, \mathcal{F}_{t}, \nu\right)$ and an increasing filtration $\mathcal{F}_{t} \subset \mathcal{F}, t \in[0, T]$. The CAT bond is well characterized by:

- A doubly stochastic Poisson process (see Bremaud 1981) $M_{s}(s \in[0, T])$ describing the flow of natural events of a given type in the region. The intensity of this process is assumed to be a predictable bounded process $m_{s}$.

- The losses $\left\{X_{i}\right\}_{i \in \mathbb{N}}$, which are independent and identically distributed with $F(x)=\mathbf{P}\left\{X_{i}<x\right\}$. Moreover, $X$ and $M$ are independent.

- The aggregate loss process $L_{t}=\sum_{i=1}^{M_{t}} X_{i}$.

- A pogressive process of discounting rates $r$. We assume the process is continuous a.e. This process describes the value at time $s$ of USD 1 paid at time $t>s$ by

$$
\exp (-R(s, t))=\exp \left(-\int_{s}^{t} r(\xi) d \xi\right) .
$$

- The maturity time $T$ and threshold level $D$.

- The threshold time $\tau=\inf \left\{t: L_{t} \geq D\right\}$, which is the moment when the aggregated loss $L_{t}$ exceeds the value $D$.

- In the case of a zero-coupon bond: payment of a certain (random) amount $Z$ at maturity time $T$ contingent on threshold time $\tau>T$.

- In the case of a coupon bond: payment of the principal (face value) at maturity time $T$ contingent on threshold time $\tau>T$ and coupon payments $C_{t}$ which stop immediately at $\tau$ (in general a process which is predictable and continuous on $[0, T]$ ).

Now define a new process $N_{t}=\mathbf{1}_{\left\{L_{t} \geq D\right\}}$. Baryshnikov et al. (1998) in Proposition 2 show that this is also a doubly stochastic Poisson process with the intensity

$$
\lambda_{s}=m_{s}\left(1-F\left(D-L_{s}\right)\right) \mathbf{1}_{\left\{L_{s}<D\right\}} .
$$


Let us consider a bond with the payment of a certain amount $Z$ at maturity time $T$ contingent on threshold time $\tau>T$, which is in fact a zero-coupon CAT bond. Define the process $Z_{s}=\mathbf{E}\left(Z \mid \mathcal{F}_{s}\right)$. The condition required is that $Z_{s}$ is a predictable process, which can be interpreted to mean that the payment at maturity is not directly linked to the occurrence and timing of the threshold. The amount $Z$ can be e.g. the principal plus interest which is usually defined as a fixed percentage over LIBOR. We obtain the following result.

THEOREM 2.1. The non-arbitrage price of the zero-coupon CAT bond associated with the threshold $D$, the catastrophic flow $M_{s}$, and the distribution function $F$ of the incurred losses, paying $Z$ at maturity, is given by

$$
V_{t}=\mathbf{E}\left(Z_{t} e^{-R(t, T)}\left[1-\int_{t}^{T} m_{s}\left[1-F\left(D-L_{s}\right)\right] \mathbf{1}_{\left\{L_{s}<D\right\}} d s\right] \mid \mathcal{F}_{t}\right) .
$$

Proof. Clearly the price of a CAT bond paying $Z$ at maturity at time $t<\tau$ is

$$
V_{t}=\mathbf{E}\left(Z e^{-R(t, T)}\left(1-N_{T}\right) \mid \mathcal{F}_{t}\right) .
$$

Representing $N_{T}$ as $\int_{t}^{T} d N_{s}$ one arrives at the expression

$$
V_{t}=\mathbf{E}\left(Z e^{-R(t, T)}\left(1-\int_{t}^{T} d N_{s}\right) \mid \mathcal{F}_{t}\right)
$$

From the definition of a doubly stochastic Poisson process (see Bremaud, 1981) we have

$$
V_{t}=\mathbf{E}\left(Z e^{-R(t, T)}\left(1-\int_{t}^{T} \lambda_{s} d s\right) \mid \mathcal{F}_{t}\right) .
$$

Now we apply (1) to get the assertion.

We note that Baryshnikov et al. (1998) in p. 3.2 of their article presented a price of the threshold bond paying $Z$ at maturity, which corresponds to the case of the zero-coupon CAT bond, but their formula is incorrect.

Now, we consider a CAT bond with only coupon payments $C_{t}$ which stop immediately at $\tau$. The following theorem gives the fair CAT bond price formula for such a bond.

THEOREM 2.2. The non-arbitrage price of the CAT bond associated with the threshold $D$, the catastrophic flow $M_{s}$, and the distribution function $F$ of the incurred losses, with coupon payments $C_{s}$ which cease at threshold time $\tau$, is given by

$$
V_{t}=\mathbf{E}\left(\int_{t}^{T} e^{-R(t, s)} C_{s}\left[1-\int_{t}^{s} m_{\xi}\left[1-F\left(D-L_{\xi}\right)\right] \mathbf{1}_{\left\{L_{\xi}<D\right\}} d \xi\right] d s \mid \mathcal{F}_{t}\right) .
$$


Proof. It is easy to see that the price of a CAT bond with coupon payments $C_{t}$ to the threshold event $\tau$ is

$$
V_{t}=\mathbf{E}\left(\int_{t}^{T} e^{-R(t, s)} C_{s}\left(1-N_{s}\right) d s \mid \mathcal{F}_{t}\right) .
$$

Repeating the steps as in the proof of Theorem 2.1 we obtain the conclussion.

We note that Baryshnikov et al. (1998) (p. 3.2) studied the case of coupon payments $P_{s}$ on the threshold bond, which corresponds to the above case. In the proof of Theorem 2.2 we just apply their starting formula which can be rewritten as (2). We also remark that, unfortunately, they further use integration by parts incorrectly.

Finally, we combine Theorems 2.2 and 2.1 with $Z=$ face value (FV) in order to obtain the following fair price for the coupon CAT bond.

COROLlary 2.1. The non-arbitrage price of the coupon CAT bond associated with the threshold $D$, the catastrophic flow $M_{s}$, the distribution function $F$ of the incurred losses, paying FV at maturity, and coupon payments $C_{s}$ which cease at threshold time $\tau$, is given by

$$
\begin{aligned}
V_{t}= & \mathbf{E}\left(\mathrm{FV} e^{-R(t, T)}+\int_{t}^{T} e^{-R(t, s)}\left[C_{s}\left(1-\int_{t}^{s} m_{\xi}\left[1-F\left(D-L_{\xi}\right)\right] \mathbf{1}_{\left\{L_{\xi}<D\right\}} d \xi\right)\right.\right. \\
& \left.\left.-\mathrm{FV} e^{-R(s, T)} m_{s}\left[1-F\left(D-L_{s}\right)\right] \mathbf{1}_{\left\{L_{s}<D\right\}}\right] d s \mid \mathcal{F}_{t}\right) .
\end{aligned}
$$

In the foregoing cases (see Theorem 2.1 and Corollary 2.1) we assumed that in the case of the trigger event the principal is fully lost. However, if we would like to incorporate a partial loss in the contract it is just enough to multiply $Z$ by a proper constant.

3. Calibration of the pricing model. We conducted empirical studies for data obtained from Property Claim Services. The data (see Figure 1) concern the US market's loss amounts in USD, which occurred between 1990 and 1999 and were adjusted using the discount window borrowing rate (the discount rate refers to the simple interest rate at which depository institutions borrow from the Federal Reserve Bank of New York). Only natural perils like hurricane, tropical storm, wind, flooding, hail, tornado, snow, freezing, fire, ice and earthquake which caused damages exceeding USD $5 \mathrm{~m}$ were taken into consideration. We notice that peaks in Figure 1 mark the occurrence of Hurricane Andrew (24 August 1992) and Northridge Earthquake (17 January 1994).

In order to calibrate the pricing model we have to fit both the distribution function of the incurred losses $F$ and the process $M_{t}$ governing the flow of 
natural events. We commence with the presentation of typical claim size distributions.

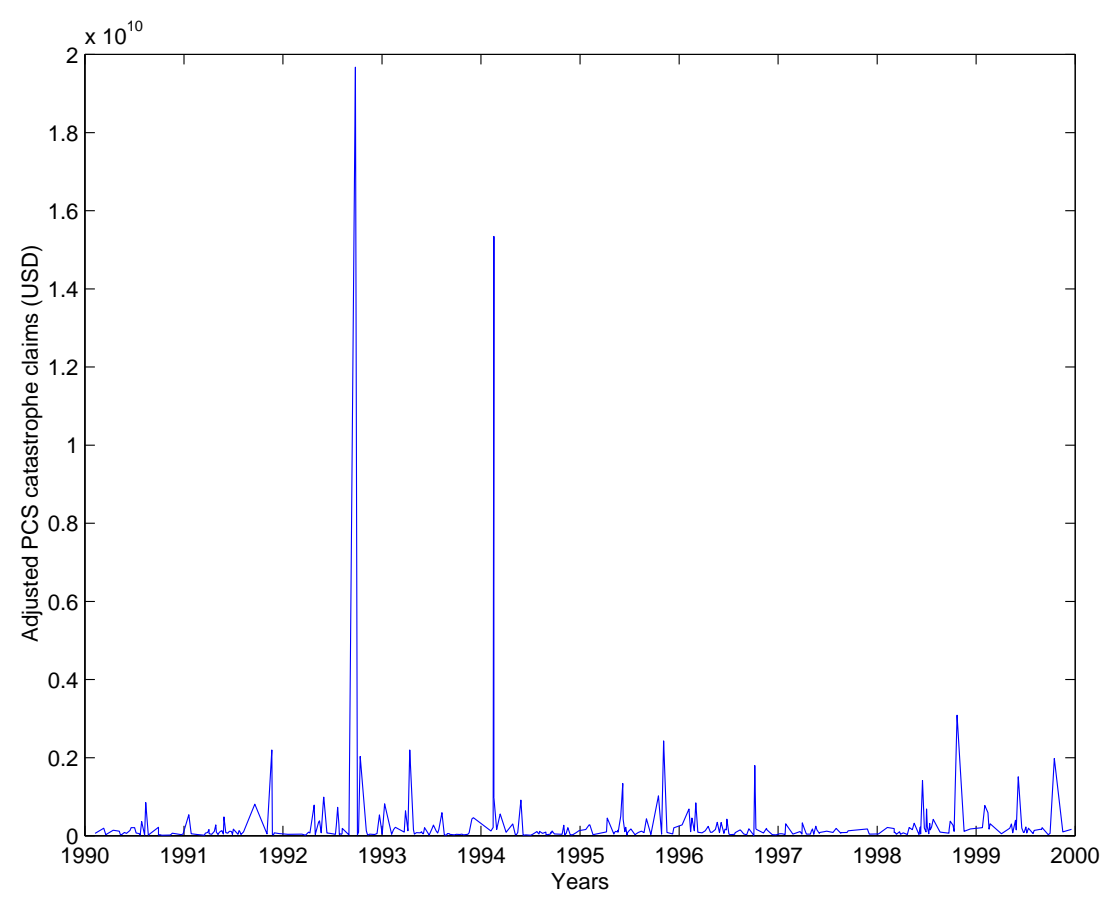

Fig. 1. Illustration of the PCS catastrophe loss data, 1990-1999

3.1. Claim amount distributions. The claim distributions, especially describing property losses, are usually heavy-tailed. In the actuarial literature, to describe such claims continuous distributions are often proposed (with the domain $\mathbb{R}_{+}$):

- lognormal distribution, with the distribution function (d.f.) given by

$$
F(x)=\Phi\left(\frac{\ln x-\mu}{\sigma}\right)=\int_{0}^{x} \frac{1}{\sqrt{2 \pi} \sigma y} e^{-\frac{1}{2}\left(\frac{\ln y-\mu}{\sigma}\right)^{2}} d y, \quad x>0, \sigma>0, \mu \in \mathbb{R},
$$

where $\Phi(x)$ is the standard normal (with mean 0 and variance 1) d.f.;

- Pareto distribution, with the d.f.

$$
F(x)=1-\left(\frac{\lambda}{\lambda+x}\right)^{\alpha}, \quad x>0, \alpha>0, \lambda>0 ;
$$

- Burr distribution, with the d.f.

$$
F(x)=1-\left(\frac{\lambda}{\lambda+x^{\tau}}\right)^{\alpha}, \quad x>0, \alpha>0, \lambda>0, \tau>0
$$


- gamma distribution, with the d.f.

$$
F(x)=\int_{0}^{x} \frac{1}{\Gamma(\alpha) \beta^{\alpha}} y^{\alpha-1} e^{-y / \beta} d y, \quad x>0, \alpha>0, \beta>0 .
$$

The choice of the distribution is very important because it influences the bond price.

3.2. Non-parametric tests. The derivation of claim size distributions from the loss data could be considered to be a separate discipline, which requires applying methods of mathematical statistics (cf. Daykin et al. 1994). The objective is to find a distribution function $F$ which fits the observed data in a satisfactory manner. The approach most frequently adopted in the actuarial literature is to find a suitable analytic expression which fits the observed data well and which is easy to handle (see e.g. Burnecki et al. 2000).

Once the distribution is selected, we must obtain parameter estimates. In what follows we use the moment and maximum likelihood estimation. The next step is to test whether the fit is adequate. This is usually done by comparing the fitted and empirical distribution functions, more precisely, by checking whether values of the fitted distribution function at sample points form a uniform distribution (cf. D'Agostino and Stephens 1986). To this end we apply the well known and not so well known non-parametric tests, namely $\chi^{2}$, Kolmogorov-Smirnov (KS), Cramer-von Mises (CM) and AndersonDarling (AD), verifying the hypothesis of uniformity (see e.g. Kukla 2000).

A very natural and well known test is the $\chi^{2}$ statistic

$$
\chi_{k}^{2}=k \sum_{i=1}^{k} \frac{\left(n_{i}-n / k\right)^{2}}{n},
$$

where $n$ is the overall number of observations and $n_{i}$ is the number of observations which fall into the interval $[(i-1) / k, i / k] \cdot \chi_{k}^{2}$ has an approximate chi-square distribution with $k-1$ degrees of freedom. In general, the better the fit, the smaller $\chi_{k}^{2}$. This test becomes more discriminating as the sample size becomes larger.

Another classical measure of fit is the Kolmogorov-Smirnov statistic

$$
D_{n}=\sup _{x \in \mathbb{R}}|\widehat{F}(x)-F(x)|,
$$

where the empirical d.f. is defined as

$$
\widehat{F}(x)=\frac{1}{n} \sum_{i=1}^{n} \mathbf{1}_{\left\{x_{i} \leq x\right\}} .
$$

The statistic $D_{n}$ measures the distance between the empirical and fitted d.f. in the supremum norm. 
The other two tests we apply are the Cramer-von Mises and AndersonDarling tests. The former uses the statistic

$$
C_{n}=n \int_{-\infty}^{\infty}(\widehat{F}(x)-F(x))^{2} d F(x)
$$

while the latter (considered to be the best within the class of tests based on empirical d.f.) uses

$$
A D=n \int_{-\infty}^{\infty} \frac{(\widehat{F}(x)-F(x))^{2}}{F(x)(1-F(x))} d F(x) .
$$

In order to interpret the results of the tests we compare them with the corresponding critical values $C_{\alpha}$ (for the same significance level $\alpha$ ). When the value of the test is less than the corresponding $C_{\alpha}$ we accept the fit as adequate (there is no reason to reject the null hypothesis). The critical values $C_{\alpha}$ of the tests given a significance level $\alpha$ (e.g. $\alpha=0.05$ ) can be found in the literature (see e.g. D'Agostino and Stephens 1986, and Stephens 1974).

3.3. Results of the fit procedure. First we studied the loss amounts. Distributions were fitted using the moment and maximum likelihood estimation. The results of the parameter estimation and test statistics are presented in Table 1. The lognormal distribution with parameters $\mu=18.4406$ and $\sigma=1.1348$ passed all tests (the corresponding test statistics are in boldface), so we chose it for further analysis.

Table 1. Parameter estimates and test statistics for the catastrophe loss amounts

\begin{tabular}{|c||c|c|c|c|}
\hline Distributions: & lognormal & Pareto & Burr & gamma \\
\hline Parameters: & $\mu=18.4406$ & $\alpha=2.3872$ & $\alpha=3.8830$ \\
& $\sigma=1.1348$ & $\lambda=3.0320 \mathrm{e}+8$ & $\begin{array}{c}\alpha=1.0891 \mathrm{e}+5 \\
\tau=0.5407\end{array}$ & $\beta=1.6348 \mathrm{e}+8$ \\
& & & \\
\hline \multicolumn{5}{|c|}{ Test values (in brackets critical values for $\alpha=0.05):$} \\
\hline$\chi^{2}(31.4104)$ & $\mathbf{3 1 . 2 1 0 5}$ & 43.1228 & 125.4035 & 63.0175 \\
$D_{n}(0.0729)$ & $\mathbf{0 . 0 4 9 4}$ & 0.1151 & 0.2362 & 0.0960 \\
$C_{n}(0.4608)$ & $\mathbf{0 . 1 8 3 9}$ & 0.8366 & 5.4508 & 0.6895 \\
$A D(2.492)$ & $\mathbf{2 . 0 2 8 3}$ & 6.7282 & 31.9213 & 10.3872 \\
\hline
\end{tabular}

Next, we fitted the doubly stochastic Poisson process governing the occurrence times of the losses. We started the analysis with the simplest case assuming the intensity $m_{s}$ is equal to a non-negative constant $m$. Studies of the quarterly numbers of losses and the interoccurrence times of the catastrophes led to the conclusion that the flow of the events may be described by the Poisson process with the daily intensity $m=0.095$. 
The parameters of the fitted model imply that the expected value of a daily loss is USD $m e^{\mu+\sigma^{2} / 2} \sim$ USD $19 \mathrm{~m}$.

4. Dynamics of the CAT bond price. In this section we present CAT bond prices for $t=0$ days, namely $V_{0}$. To this end we apply the appropriate formulae and Monte Carlo simulations. We assume for illustration purposes that the continuous discount rate $r$ equivalent to LIBOR $=2.5 \%$ is constant and equal to $\ln (1.025), T \in[90,720]$ days, $D \in \mathrm{USD}[1.71,8.55]$ bn (quarterly to $5 *$ quarterly average loss) and the principal is USD 1 .

Furthermore, in the case of the zero-coupon CAT bond we assume that the bond is priced at $3.5 \%$ over LIBOR. If there is no trigger event, the total yield is hence $6 \%$ and we put $Z=$ USD 1.06 . Figure 2 depicts the zerocoupon CAT bond values with respect to the threshold level and expiration time (cf. Theorem 2.1). We can see that the greater the expiration time, the lower the CAT bond value, and increasing the threshold level leads to greater prices. When $T=90$ days and $D=$ USD 8.55 bn the CAT bond price approaches the value USD $1.06 e^{-\ln (1.025) / 4} \sim$ USD 1.053, which corresponds to the situation when $\tau \geq T$ w.p.1.

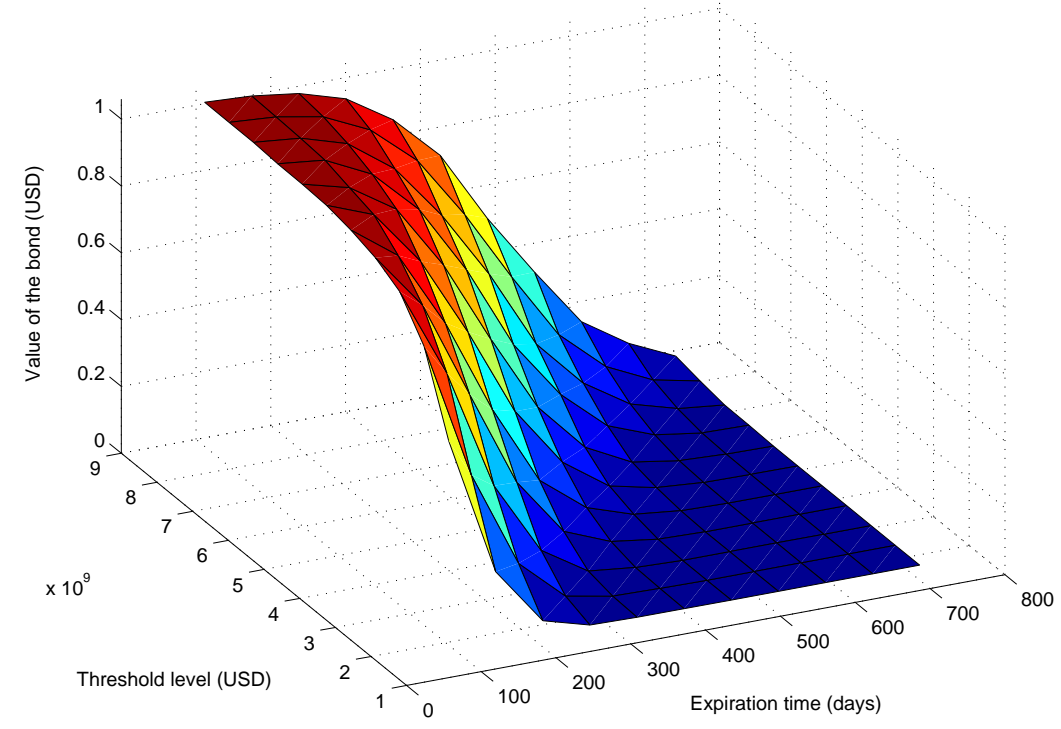

Fig. 2. The zero-coupon CAT bond price with respect to the threshold level and expiration time

Before we present the case of the coupon bond, we concentrate on the bond paying only coupons (cf. Theorem 2.2). We assume $C_{t} \equiv 0.06$. The values of $V_{0}$ are depicted in Figure 3 . We clearly see that now the situation is quite different, namely the price increases when the expiration time and 
threshold level are greater. When $D=$ USD 8.55 bn and $T=720$ days the price of the bond approaches USD $0.06 \int_{0}^{720} e^{-\ln (1.025) t / 360} d t \sim$ USD 0.08, which corresponds to the situation when $\tau \geq T$ w.p.1.

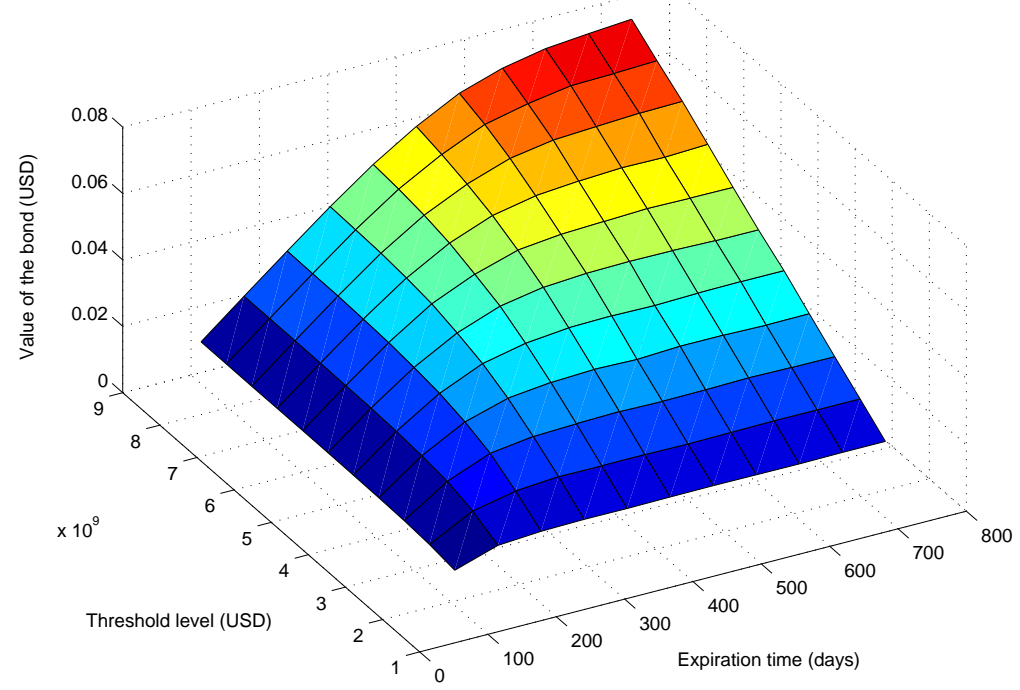

Fig. 3. The CAT bond price, for the bond paying only coupons, with respect to the threshold level and expiration time

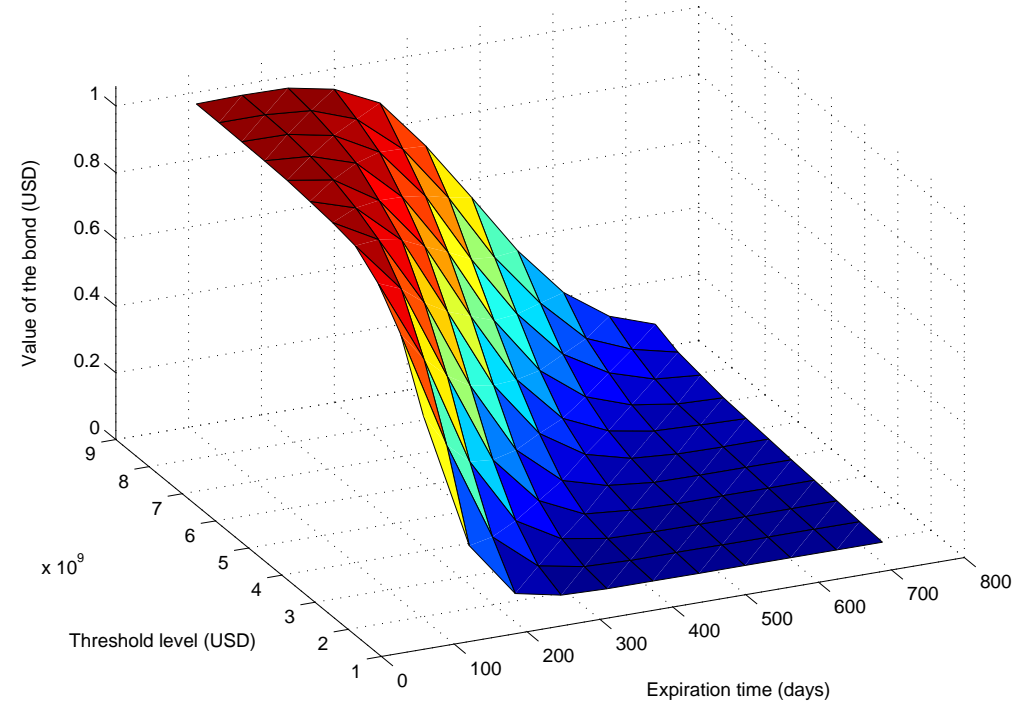

Fig. 4. The coupon CAT bond price with respect to the threshold level and expiration time 
Finally, we consider the case of the coupon CAT bond. We assume as previously that $C_{t} \equiv 0.06$. We can see the CAT bond prices in Figure 4 . The influence of the threshold level $D$ on the bond value is clear as in the cases discussed above but the effect of changing the expiration time $T$ is not straightforward. As $T$ gets bigger the chance of receiving more coupons is greater but at the same time the possibility of loosing the principal of the bond increases. In our case (see Figure 4) the price decreases with respect to the expiration time but this does not have to be always true. We also notice that the bond prices in Figure 4 are lower than the corresponding ones in Figure 2, but we must remember that in the latter case $Z=$ USD 1.06 and now we have $Z=$ USD 1 (cf. Corollary 2.1).

Acknowledgements. The first author thankfully acknowledges the support of the State Committee for Scientific Research (KBN) Grant No. PBZ-KBN 016/P03/99.

\section{References}

R. B. D'Agostino and M. A. Stephens (1986), Goodness-of-Fit Techniques, Marcel Dekker, New York.

Yu. Baryshnikov, A. Mayo and D. R. Taylor (1998), Pricing of CAT bonds, working paper (http://www.cam.wits.ac.za/mfinance/research.html).

P. Bremaud (1981), Point Processes and Queues. Martingale Dynamics, Springer, New York.

K. Burnecki, G. Kukla and R. Weron (2000), Property insurance loss distributions, Physica A 287, 269-278.

C. D. Daykin, T. Pentikainen and M. Pesonen (1994), Practical Risk Theory for Actuaries, Chapman \& Hall, London.

G. Kukla (2000), Insurance Risk Derivatives, MSc thesis, Wrocław Univ. of Technology. M. A. Stephens (1974), EDF statistics for goodness-of-fit and some comparisons, J. Amer. Statist. Assoc. 69, 730-737.

Hugo Steinhaus Center

for Stochastic Methods

Institute of Mathematics

Wrocław University of Technology

Wybrzeże Wyspiańskiego 27

50-370 Wrocław, Poland

E-mail: burnecki@im.pwr.wroc.pl

kukla@im.pwr.wroc.pl 